\title{
Tyrosinase Peptide
}

National Cancer Institute

\section{Source}

National Cancer Institute. Tyrosinase Peptide. NCI Thesaurus. Code C2401.

One of a number of recombinant peptides consisting of amino acid residues of the enzyme tyrosinase, a protein frequently expressed by melanoma cells. Vaccination with tyrosinase peptide may stimulate cytotoxic $\mathrm{T}$ lymphocyte response against tyrosinaseexpressing tumor cells, resulting in decreased tumor growth. (NCI04) 